\title{
Multi-dimensional modeling of solar cells with electromagnetic and carrier transport calculations
}

\author{
Xiaofeng Li ${ }^{*}$, Nicholas P. Hylton, Vincenzo Giannini, Kan-Hua Lee, Ned J. Ekins-Daukes and \\ Stefan A. Maier \\ Blackett Laboratory, Department of Physics, Imperial College London, London SW7 2AZ, UK
}

\begin{abstract}
We present a multi-dimensional model for comprehensive simulations of solar cells (SCs), considering both electromagnetic and electronic properties. Typical homojunction and heterojunction gallium arsenide SCs were simulated in different spatial dimensions. When considering one-dimensional problems, the model performs carrier transport calculations following a Beer-Lambert optical absorption approximation. We show that the results of such simulations exhibit excellent agreement with the standard PC1D one-dimensional photovoltaic simulation. Photonic and plasmonic attempts to enhance SC efficiency demand comprehensive electromagnetic calculations to be undertaken in order to acquire accurate carrier generation profiles in two and three-dimensional systems. Our model provides complete spectral and spatial information of typical optical and electronic behavior. Furthermore, our approach permits the detailed investigation of complex systems, including plasmonic SCs, which cannot be simulated using low-dimensional modeling tools. We present the results of numerical simulations of an optically thin plasmonic gallium arsenide SC and observe improved device performance arising from the application of plasmonic nanostructures, which agree well with previous experimental findings. Copyright (C) 2012 John Wiley \& Sons, Ltd.
\end{abstract}

\section{KEYWORDS}

photovoltaics; solar cells; carrier transport; multi-dimensional modeling; surface plasmons

* Correspondence

Xiaofeng Li, Blackett Laboratory, Department of Physics, Imperial College London, London SW7 2AZ, UK.

E-mail: x.li@imperial.ac.uk

Received 26 August 2011; Revised 24 November 2011; Accepted 5 December 2011

\section{INTRODUCTION}

Accurate two-dimensional (2D) and three-dimensional (3D) modeling of solar cells (SCs) are becoming increasingly important due to the advent of low-cost nanofabrication that may enable advanced highly efficient photovoltaic devices. The standard and simplest way to simulate an SC is to use analytical expressions describing the carrier transport characteristics within the device [1]; however, the applicability of such analytical expressions is limited to simple onedimensional (1D) problems. A more thorough approach is to numerically solve the carrier transport equations for user-defined device configurations [2]. Nowadays, there are a number of simulation approaches or software available for the modeling of SCs, including PC1D, DESSIS, SCAPS, AFORS-HET, AMPS, and so on. [3-8]. However, these packages mainly work in one or two dimensions $(1 \mathrm{D}, 2 \mathrm{D})$, and carrier generation has seldom been treated thoroughly; instead, low-dimensional approximations (e.g., the Beer-Lambert and depletion approximations) have been commonly used.

For ordinary SCs with no obvious transverse dependence (i.e., along the direction parallel to the junction interfaces), 1D simulations can be sufficiently accurate; otherwise, higher-dimensional (2D or 3D) simulations must be used. For example, the incorporation of surface plasmons (SPs), the eigenmodes of metallodielectric structures [9-11], are of interest in PV devices to realize efficient light absorption in thin-film SCs [12,13]. In these devices, plasmonic nanostructures can be integrated to obtain a long optical path without changing the original geometry of the device. Plasmonic solar cells (PSCs) and photodetectors based on organic/inorganic materials employing various nanostructures have been proposed and shown improved optical absorption and light-conversion capability [12-24].For the design of PSCs to be optimized, the 
simulation normally has to be realized in $3 \mathrm{D}$, because the spatial pattern of the optical mode (i.e., carrier generation) is modified strongly by the excited resonant SP modes. For this reason, carrier generation profiles cannot be predicted directly from the traditional optical approximation; instead, a thorough electromagnetic (EM) calculation is required.

We have recently reported 3D simulations of PSCs by bridging EM and carrier transport calculations [25], which allows us to examine the SC performance [e.g., external quantum efficiency (EQE), short-circuit current density $J_{\text {sc }}$, open-circuit voltage $V_{\mathrm{oc}}$, fill factor $F F$, and lightconversion efficiency $\eta$ ] rather than focusing solely on optical absorption. In fact, even for conventional nonplasmonic SCs, such a complete modeling scheme has seldom been comprehensively addressed, even for lowdimensional cases (1D or 2D) [1-8]. In this paper, we introduce in detail our methodology for modeling SCs considering both the EM and electronic response in spatial dimensions from $1 \mathrm{D}$ to 3D. The mathematical background and our treatment will be introduced in Section 2 . Section 3 presents a comparison between our model and PC1D, which were both used to simulate a 1D homojunction gallium arsenide (GaAs) SC. In Section 4, 2D modeling has been conducted for both homojunction and heterojunction GaAs SCs, and comparisons of our results with both PC1D and typical experiments are given. In Section 5, an optically thin GaAs SC with plasmonic nanoparticle design was simulated in $3 \mathrm{D}$ and the resulting improvement of both the optical and electronic performance of SCs is discussed. Finally, a summary will be given in Section 6.

\section{MODEL DESCRIPTION}

Our model aims to perform multi-dimensional modeling of SCs by using Comsol Multiphysics on the basis of the finite-element method [26]. In 1D, it performs a carrier transport calculation where the carrier generation profile is obtained from the Beer-Lambert law [1]. In 2D and $3 \mathrm{D}$, instead of using this optical approximation, the exact EM response is calculated before carrying out an electronic calculation, so that accurate optical and electronic characteristics of the SCs can be obtained. In our model, the radio-frequency module of Comsol is used for simulations of the optical response by considering one cell surrounded by Floquet-Bloch boundaries and perfectly matched layers; the electronic part consists of three modules, that is, two convection-diffusion modules describing carrier transport under the effects of carrier diffusion, drift, generation and recombination, and one electrostatic potential module based on Poisson's equation. Because the EM calculation, which is based on Maxwell's equations, has been extensively discussed [10,15,16,21], we solely concentrate on the $3 \mathrm{D}$ mathematical model describing the electronic response of the device. The lower-dimensional cases (1D and 2D) can be easily derived from the 3D model by removing the corresponding spatial dependences.

\subsection{Carrier transport equations in $3 D$}

To develop a general model for the simulation of SCs with various system configurations (i.e., spatial dimension, material composition, etc.), we employ the following equations, which consist of 3D carrier transport and Poisson's equations and consider the gradients of intrinsic material parameters around the heterojunction interfaces $[1,27]$ :

$$
\begin{gathered}
\nabla\left[-D_{n} \nabla n+n \mu_{n}\left(\nabla \Phi+\frac{\nabla \chi}{q}+\frac{K_{\mathrm{B}} T}{q} \nabla \ln N_{\mathrm{c}}\right)\right](1 \mathrm{a}) \\
=g(x, y, z, \lambda)-U \\
\nabla\left[-D_{p} \nabla p-p \mu_{p}\left(\nabla \Phi+\frac{\nabla \chi}{q}+\frac{\nabla E_{\mathrm{g}}}{q}-\frac{K_{\mathrm{B}} T}{q} \nabla \ln N_{\mathrm{v}}\right)\right](1 \mathrm{~b}) \\
=g(x, y, z, \lambda)-U \\
\nabla^{2} \Phi=\frac{q}{\varepsilon_{0} \varepsilon_{\mathrm{r}}}(n-p-C)
\end{gathered}
$$

where $n(p)$ is the electron (hole) concentration, $D_{n}=$ $\mu_{n} K_{\mathrm{B}} T / q \quad\left(D_{p}=\mu_{p} K_{\mathrm{B}} T / q\right)$ the electron (hole) diffusion coefficient, $\mu_{n}\left(\mu_{p}\right)$ the electron (hole) mobility, $K_{\mathrm{B}}$ the Boltzmann's constant, $T=300 \mathrm{~K}$ the operating temperature, $q$ the electronic charge, and $\Phi$ the electrostatic potential (electrostatic field $F=-\nabla \Phi) . \chi$ is the electron affinity, $E_{\mathrm{g}}$ the semiconductor bandgap, $N_{\mathrm{c}}=\left[m_{\mathrm{c}} K_{\mathrm{B}} T /\left(2 \pi \hbar^{2}\right)\right]^{1.5}$ $\left(N_{\mathrm{v}}=\left[m_{\mathrm{v}} K_{\mathrm{B}} T /\left(2 \pi \hbar^{2}\right)\right]^{1.5}\right)$ the effective conduction (valence) band density of states, $m_{\mathrm{c}}\left(m_{\mathrm{v}}\right)$ the conduction (valence) band effective mass, $\hbar$ the reduced Planck constant, $g$ the carrier generation rate, $\lambda$ the wavelength, and $U$ the carrier recombination rate. Finally, $\varepsilon_{0}\left(\varepsilon_{\mathrm{r}}\right)$ is the vacuum permittivity (relative permittivity of the material) and $C=N_{\mathrm{D}}-N_{\mathrm{A}}$ the impurity concentration defined as the sum of the concentrations of ionized donors $N_{\mathrm{D}}$ and acceptors $N_{\mathrm{A}}$, including the signs of the compensated charges.

$U$ includes contributions from Shockley-Read-Hall, radiative, and Auger recombinations

$$
\begin{gathered}
U=U_{\mathrm{SRH}}+U_{\text {rad }}+U_{\text {aug }} \\
U_{\mathrm{SRH}}=\frac{n p-n_{i}^{2}}{\tau_{n}\left(p+p_{\mathrm{t}}\right)+\tau_{p}\left(n+n_{\mathrm{t}}\right)} \\
U_{\mathrm{rad}}=B_{\mathrm{rad}}\left(n p-n_{i}^{2}\right) \\
U_{\mathrm{aug}}=\left(C_{n} n+C_{p} p\right)\left(n p-n_{i}^{2}\right)
\end{gathered}
$$

where $\tau_{n}\left(\tau_{p}\right)$ is the electron (hole) lifetime, $n_{\mathrm{t}}\left(p_{\mathrm{t}}\right)$ the electron (hole) concentration of the trap state (the strongest $U_{\mathrm{SRH}}$ occurs when $\left.n_{\mathrm{t}}=p_{\mathrm{t}}=n_{i}[2]\right), n_{i}=\left[N_{\mathrm{c}} N_{\mathrm{v}} \exp \left(-q E_{\mathrm{g}} / K_{\mathrm{B}} \mathrm{T}\right)\right]^{1 / 2}$ the intrinsic carrier concentration, $B_{\mathrm{rad}}$ the coefficient of bimolecular radiative recombination, and $C_{n}\left(C_{p}\right)$ the electron (hole) Auger coefficient. 
The frequency-dependent carrier generation rate can be estimated directly from the Beer-Lambert law in 1D. However, more accurate results can be obtained through an exact EM calculation for higher-dimensional cases:

$$
g(x, y, z, \lambda)=\alpha(x, y, z, \lambda) b_{\mathrm{s}}(\lambda) P_{\mathrm{s}}(x, y, z, \lambda)
$$

where $\left.\alpha=4 \pi n_{\text {imag }} / \lambda\right)$ is the extinction coefficient of the photoactive material, $n_{\text {imag }}$ the imaginary part of its refractive index, and $b_{\mathrm{s}}$ the solar incident photon flux (AM1.5 [28]). The power flow, $P_{\mathrm{s}}$, is a key factor that connects the EM and electronic parts. It can be derived from the Poynting vector: and $p_{\text {init }}$, can be obtained by applying the neutral charge condition in each layer together with the $n_{i}$ expression [29]:

$$
\begin{gathered}
n_{\text {init }}-p_{\text {init }}-C=0 \\
n_{\text {init }} p_{\text {init }}=n_{i}^{2}
\end{gathered}
$$

Therefore, $n_{\text {init }}$ and $p_{\text {init }}$ can be expressed as

$$
\begin{aligned}
& n_{\text {init }}=\frac{1}{2}\left(\sqrt{C^{2}+4 n_{i}^{2}}+C\right) \\
& p_{\text {init }}=\frac{1}{2}\left(\sqrt{C^{2}+4 n_{i}^{2}}-C\right)
\end{aligned}
$$

$$
P_{\mathrm{s}}(x, y, z, \lambda)=\sqrt{\left|P_{o x}(x, y, z, \lambda)\right|^{2}+\left|P_{o y}(x, y, z, \lambda)\right|^{2}+\left|P_{o z}(x, y, z, \lambda)\right|^{2}}
$$

where the flux components in the $x, y$, and $z$ directions are, respectively

$$
\begin{aligned}
& P_{o x}(x, y, z, \lambda)=\frac{1}{2} \mathfrak{R}\left(E_{y} H_{z}^{*}-E_{z} H_{y}^{*}\right) \\
& P_{o y}(x, y, z, \lambda)=\frac{1}{2} \mathfrak{R}\left(E_{z} H_{x}^{*}-E_{x} H_{z}^{*}\right) \\
& P_{o z}(x, y, z, \lambda)=\frac{1}{2} \mathfrak{R}\left(E_{x} H_{y}^{*}-E_{y} H_{x}^{*}\right)
\end{aligned}
$$

Here, $E(H)$ is the frequency and spatially dependent electric (magnetic) field yielded by external light injection, and the symbols " $R$ " and "*" are the operators to take the real part and the complex conjugation, respectively. In our carrier transport module, $P_{\mathrm{s}}$ is used as a dimensionless parameter that reflects the relative power distribution in the device under a unit incident power density. The actual photon flux under solar illumination is obtained by including $b_{\mathrm{s}}(\lambda)$ as in Equation (4). Because we aim to obtain exact solutions of Maxwell's equations, all optical mechanisms (e.g., surface reflection, light interference, absorption, etc.) determining the field distribution have been automatically taken into account, enabling us to obtain carrier generation profiles for the subsequent electronic calculation. With the consideration of the detailed intensity and spatial dependence of the incident light, our model can be used to simulate the systems under more complicated configurations (e.g., with an external optical concentrator or plasmonic nanostructures), where the 3D diffusion and generation of carriers can be modeled accurately.

\subsection{Initial conditions}

In the case of 2D and 3D modeling, the initial conditions of $n, p$, and $\Phi$ play an important role in affecting the calculation accuracy and efficiency. Under predefined doping profiles, a good estimation of the initial carrier profiles, that is, $n_{\text {init }}$
The initial electrostatic potential, $\Phi_{\text {init }}$, can be estimated for both Ohmic and Schottky contacts. For an Ohmic contact, $\Phi_{\text {init }}$ has the following form [29]:

$$
\Phi_{\text {init }}=\frac{K_{B} T}{q} \arcsin h\left(\frac{C}{2 n_{i}}\right)
$$

This equation gives a good prediction of the initial $\Phi$ profile in homojunction SCs, for example, GaAs SCs composed of $\mathrm{p}$ and $\mathrm{n}$-GaAs layers. However, it is not valid for those with more complex material compositions, for example, heterojunction GaAs SCs composed of a p-AlGaAs window (doping concentration: $N_{\mathrm{Aw}}$; AlGaAs: aluminum gallium arsenide), a heavily doped p-GaAs $\left(N_{\mathrm{A} 1}\right)$ emitter, a lightly doped $\mathrm{n}$-GaAs $\left(N_{\mathrm{D} 1}\right)$ base, and an n-AlGaAs $\left(N_{\text {DB }}\right)$ back surface field (BSF) layer. The fundamental reason is that Equation (9) only considers the doping effect but neglects the potential change in the heterojunction interfaces [1,27]. If a heterojunction SC is considered in our model, the built-in potentials between all junctions are calculated so that the relative potential in each layer can be determined. Taking a typical heterojunction GaAs SC as the example, the built-in potentials $\left(V_{P P}, V_{P N}\right.$, and $\left.V_{N N}\right)$ for the p-AlGaAs/p-GaAs, p-GaAs/n-GaAs, and n-GaAs/ n-AlGaAs junctions can be written as [30]

$$
\begin{aligned}
& V_{P P}=\frac{1}{q}\left[\Delta E_{\mathrm{c}}+K_{\mathrm{B}} T \ln \left(\frac{N_{\mathrm{v} 1} N_{\mathrm{AW}}}{N_{\mathrm{A} 1} N_{\mathrm{vW}}}\right)\right] \\
& V_{P N}=\frac{1}{q}\left[\Delta E_{\mathrm{g} 1}+K_{\mathrm{B}} T \ln \left(\frac{N_{\mathrm{A} 1} N_{\mathrm{D} 1}}{N_{\mathrm{c} 1} N_{\mathrm{v} 1}}\right)\right] \\
& V_{N N}=\frac{1}{q}\left[\Delta E_{\mathrm{v}}+K_{\mathrm{B}} T \ln \left(\frac{N_{\mathrm{c} 1} N_{\mathrm{DB}}}{N_{\mathrm{D} 1} N_{\mathrm{cB}}}\right)\right]
\end{aligned}
$$

respectively, where $N_{\mathrm{c} \#}$ and $N_{\mathrm{v} \# \text { are doping concentrations }}$ for materials in the layer indicated by the subscript "\#" ("1" for GaAs, " $W$ " for window, " $B$ " for BSF). The conduction (for PP heterojunction) and valence (for NN heterojunction) band offsets ( $\Delta E_{\mathrm{c}}$ and $\left.\Delta E_{\mathrm{v}}\right)$ can be obtained 
according to the "60:40 rule" that is particularly valid for AlGaAs/AlAs interfaces [31], hence

$$
\begin{aligned}
& \Delta E_{\mathrm{c}}=0.6\left(E_{\mathrm{gW}}-E_{\mathrm{g} 1}\right) \\
& \Delta E_{\mathrm{v}}=0.4\left(E_{\mathrm{gB}}-E_{\mathrm{g} 1}\right)
\end{aligned}
$$

where $E_{\mathrm{g} 1}\left(E_{\mathrm{gW}}, E_{\mathrm{gB}}\right)$ is the bandgap energy in GaAs (window, BSF) layer. In many other cases, Anderson's rule can be used to determine the values of $\Delta E_{\mathrm{c}}$ and $\Delta E_{\mathrm{v}}$ [32].

\subsection{Boundary conditions}

Periodic boundary conditions were used in the transverse directions for both carrier diffusion and electrostatic potential modules. For SCs without transverse dependences, a short period $(\Lambda)$ can be used; however, when surfacetextured or plasmonic SCs are considered, the dimensions of the texturing or metallic particles dictate $\Lambda$.

Along the direction perpendicular to the junction interfaces, surface recombination conditions are used for the carrier diffusion modules. The minority surface recombination conditions at the exterior surfaces of the $\mathrm{p}$ and n-regions of the SCs are as follows:

$$
\begin{gathered}
D_{n} \frac{d n}{d z}=S_{n}\left(n-n_{\text {int }}\right) \\
D_{p} \frac{d p}{d z}=-S_{p}\left(p-p_{\text {int }}\right)
\end{gathered}
$$

These conditions can be realized by introducing the corresponding "inward flux" in the convection-diffusion modules in Comsol [26].

Finally, for Poisson's equation under a forward electric bias $(V)$, we assume that the value of $\Phi$ at the device surface connecting the cathode port is unchanged, whereas the opposite end is increased by $V$ from its initial value.

\subsection{Performance evaluation}

The solutions of Equations (1a)-(1c) enable the examination of the electronic response of the SCs. The spatially and frequency-dependent photocurrent volume density contributed from electrons and holes can then be obtained from the following:

$$
\begin{aligned}
& j_{n}=q D_{n} \nabla n+n \mu_{n}\left(q F-\nabla \chi-K_{\mathrm{B}} T \nabla \ln N_{\mathrm{c}}\right) \\
& j_{p}=-q D_{p} \nabla p+p \mu_{p}\left(q F-\nabla \chi-\nabla E_{\mathrm{g}}+K_{\mathrm{B}} T \nabla \ln N_{\mathrm{v}}\right)
\end{aligned}
$$

The corresponding short-circuit current density $j_{\mathrm{sc}}(\lambda)$ is given by the averaged photocurrent at the surface of the SCs (both surfaces give the same $j_{\mathrm{sc}}$ value)
$j_{\mathrm{sc}}(\lambda)=\frac{1}{\Lambda^{2}} \int_{-\Lambda / 2}^{\Lambda / 2} \int_{-\Lambda / 2}^{\Lambda / 2}\left|j_{n}(x, y, L, \lambda)+j_{p}(x, y, L, \lambda)\right| d x d y$

where $L$ is the overall thickness of the photoactive layers. It should be noted that the terms describing the heterojunction properties in Equations (13a) and (13b) can be removed for the calculation of $j_{\mathrm{sc}}(\lambda)$. The frequency-dependent EQE can be defined as [1]

$$
E Q E(\lambda)=j_{s c}(\lambda) / q b_{s}(\lambda)
$$

The overall short-circuit current density $J_{\mathrm{sc}}$ can then be achieved through spectral integration of $j_{\mathrm{sc}}(\lambda): J_{\mathrm{sc}}=\int j_{\mathrm{sc}}(\lambda)$ $\mathrm{d} \lambda$. With the application of forward electric bias to the SCs and neglecting light generation, the dark current density $J_{\mathrm{d}}(V)$ can also be calculated using the electronic modules. Considering device resistances, the currentvoltage $(I-V)$ response of the SCs can finally be written as [1]

$$
J(V)=J_{\mathrm{sc}}-J_{\mathrm{d}}(V)-\frac{V+J(V) R_{\mathrm{s}}}{R_{\mathrm{sh}}}
$$

where $R_{\mathrm{S}}$ and $R_{\mathrm{sh}}$ are the series and shunt resistances in $\Omega \mathrm{cm}^{2}$, respectively. From the $I-V$ curve, performance parameters including open-circuit voltage $V_{\mathrm{oc}}$, maximum output power density $P_{\max }$, fill factor $\left(F F=P_{\max } / J_{\mathrm{sc}} V_{\mathrm{oc}}\right)$, and light-conversion efficiency $\left(\eta=P_{\text {max }} / P_{\text {sun }}\right.$, where $P_{\text {sun }}$ is the overall incident power density) can all be obtained.

In this study, both homojunction and heterojunction GaAs SCs with various system setups were simulated using our model. In the simulations outlined in the following parts, the parameter values listed in Table I [33-39] were used, unless otherwise indicated.

\section{1D MODELING CONSIDERING ELECTRONIC RESPONSE}

We treat first the 1D case, where the electronic response of a device is determined by solving the $1 \mathrm{D}$ carrier transport equations using a carrier generation profile estimated from the Beer-Lambert law. In this section, we mainly focus on the comparison between our 1D model and PC1D so that the basic physics and numerical treatment used in our model can be verified.

Plotted in Figure 1 are simulated EQE and $I-V$ curves of the homojunction GaAs SC shown in the inset of Figure 1 (a2). The direction of the solar illumination is from the p-type GaAs emitter, with surface power reflectivity assumed to be $5 \%$ [with anti-reflection coatings (ARCs)]. As evidenced by Figure 1, the EQE and $I-V$ performance calculated from PC1D and our 1D model are almost identical. The results show that the light-conversion capability of the simulated device is very low (i.e., $J_{\mathrm{sc}} \sim 13.9 \mathrm{~mA} / \mathrm{cm}^{2}$ ) despite the use of a thick $(3.5 \mu \mathrm{m}) \mathrm{GaAs}$ active layer. The primary reason 
Table I. Typical parameters of GaAs SCs [33-39].

\begin{tabular}{|c|c|c|c|c|}
\hline & Window & Emitter & Base & BSF \\
\hline Material & $\mathrm{p}-\mathrm{Al}_{0.8} \mathrm{Ga}_{0.2} \mathrm{As}$ & $\mathrm{p}$-GaAs & n-GaAs & $\mathrm{n}-\mathrm{Al}_{0.3} \mathrm{Ga}_{0.7} \mathrm{As}$ \\
\hline Thickness (nm) & 30 & 500 & 3000 & 500 \\
\hline Doping $\left(\mathrm{cm}^{-3}\right)$ & $1 \times 10^{18}$ & $4 \times 10^{18}$ & $2 \times 10^{17}$ & $1 \times 10^{18}$ \\
\hline Index & [38] & [33] & [33] & [38] \\
\hline$\varepsilon_{\mathrm{r}}$ & 10 & 11 & 11 & 9 \\
\hline$E_{\mathrm{g}}(\mathrm{eV})$ & 2.07 & 1.424 & 1.424 & 1.82 \\
\hline$\chi(\mathrm{eV})$ & 3.53 & 4.07 & 4.07 & 3.72 \\
\hline$m_{\mathrm{c}}\left(m_{0}\right)$ & 0.738 & 0.067 & 0.067 & 0.089 \\
\hline$m_{v}\left(m_{0}\right)$ & 0.71 & 0.572 & 0.572 & 0.589 \\
\hline$\mu_{n}\left(\mathrm{~cm}^{2} \mathrm{~N} / \mathrm{s}\right)$ & 200 & 1100 & 4000 & 1600 \\
\hline$\mu_{p}\left(\mathrm{~cm}^{2} \mathrm{~N} / \mathrm{s}\right)$ & 30 & 200 & 200 & 30 \\
\hline$\tau_{n}(\mathrm{~ns})$ & 0.005 & 4 & 4 & 4 \\
\hline$\tau_{p}(\mathrm{~ns})$ & 10 & 10 & 10 & 0.1 \\
\hline$B_{\text {rad }}\left(\mathrm{cm}^{3} / \mathrm{s}\right)$ & $1 \times 10^{-10}$ & $7.2 \times 10^{-10}$ & $7.2 \times 10^{-10}$ & $1 \times 10^{-10}$ \\
\hline$C_{n}\left(\mathrm{~cm}^{6} / \mathrm{s}\right)$ & $1 \times 10^{-31}$ & $1 \times 10^{-30}$ & $1 \times 10^{-30}$ & $1 \times 10^{-31}$ \\
\hline$C_{p}\left(\mathrm{~cm}^{6} / \mathrm{s}\right)$ & $1 \times 10^{-31}$ & $1 \times 10^{-30}$ & $1 \times 10^{-30}$ & $1 \times 10^{-31}$ \\
\hline \multicolumn{5}{|c|}{ Other Parameters } \\
\hline $\mathrm{MgF}_{2}$ index & 1.38 & & $S_{n}(\mathrm{~cm} / \mathrm{s})$ & $5 \times 10^{6}$ \\
\hline ZnS index & [35] & & $S_{p}(\mathrm{~cm} / \mathrm{s})$ & $5 \times 10^{3}$ \\
\hline
\end{tabular}

BSF, back surface field.
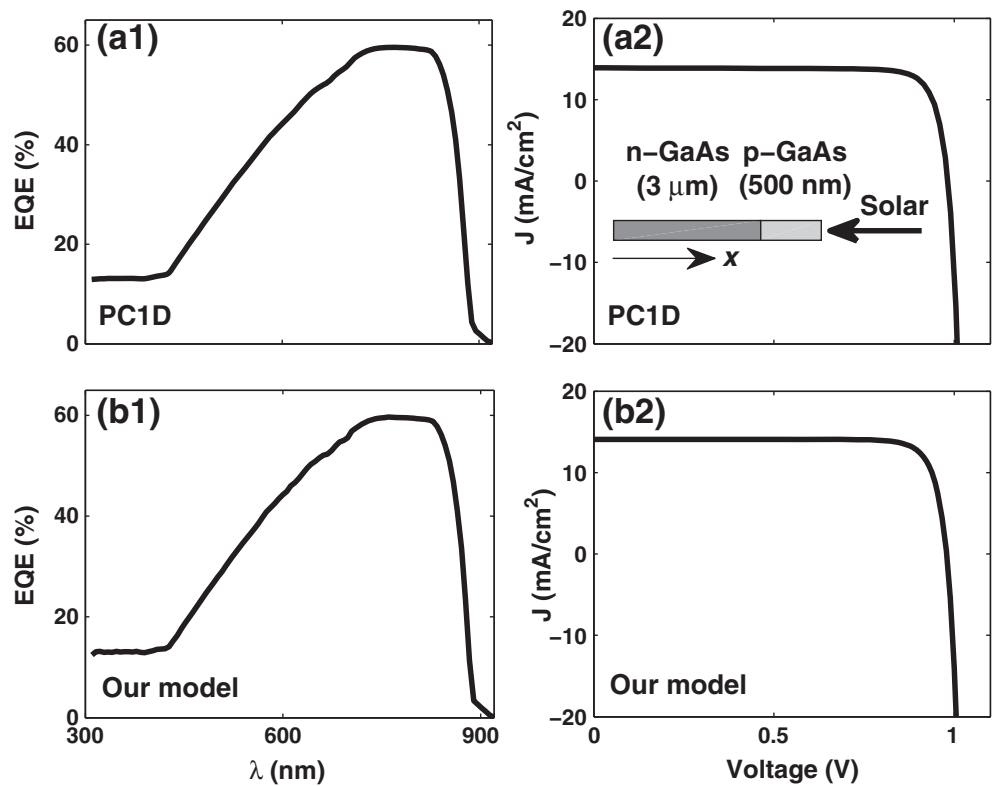

Figure 1. External quantum efficiency (EQE) spectra (a1) and (b1) and $I-V$ response (a2) and (b2) of a GaAs solar cell under homojunction P/N configuration. The results were obtained from one-dimensional (1D) electronic calculations using PC1D (a1) and (a2) and our model (b1) and (b2). The device schematic with surface reflectivity assumed to be $5 \%$ is inserted into (a2).

for this is that no window layer was employed to prohibit the diffusion of minority carriers to the surface, resulting in a large photocurrent loss due to strong surface recombination [1]. The calculation of dark current also provides information on $V_{\mathrm{oc}}$, which we found to be around $1 \mathrm{~V}$, as shown by Figure 1(a2 and b2). Further comparisons on spatial and spectral dependences of typical optical and electronic parameters were also performed. Figure 2 illustrates one example with $\lambda=600 \mathrm{~nm}$, where spatial profiles of $n, p, \Phi$, $F, g$, and $U$ have been given. A strong built-in electric field was observed in the depletion region, and the built-in voltage of the P/N GaAs junction is about $1.38 \mathrm{~V}$. The depletion width of the space charge region [which exhibits a low carrier recombination rate as shown by Figure 2(a4 and b4)] can also 

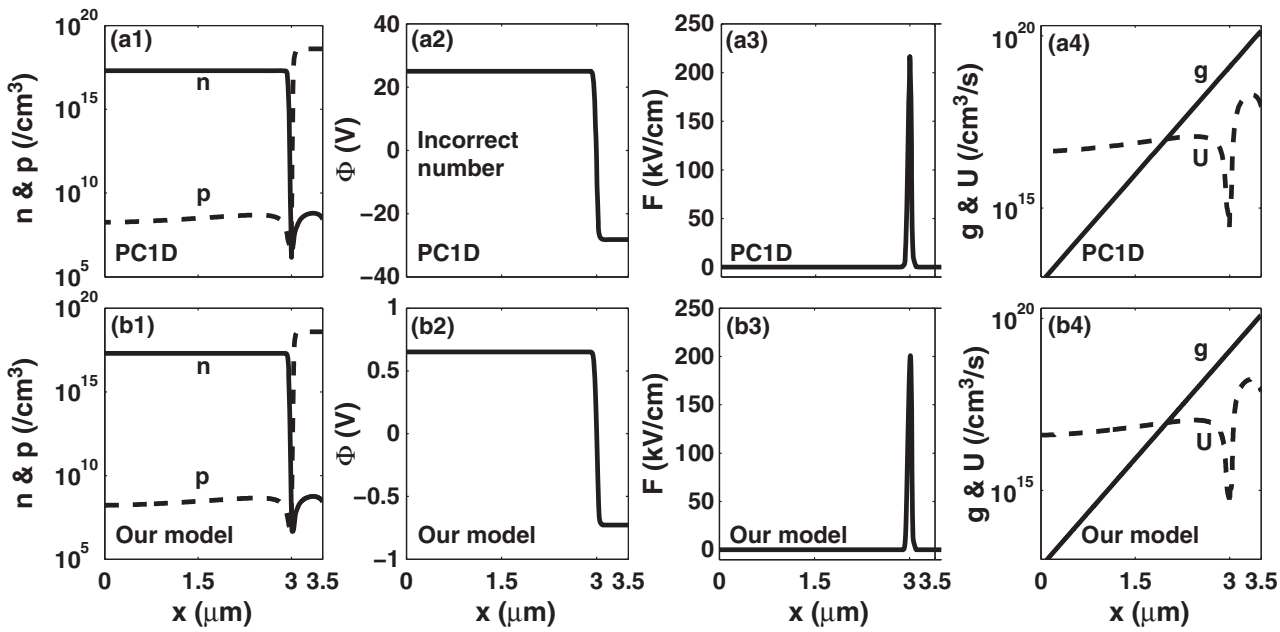

Figure 2. Profiles of $n$ and $p(a 1)$ and (b1), $\Phi(\mathrm{a} 2)$ and (b2), $F(\mathrm{a} 3)$ and (b3), and $g$ and $U(\mathrm{a} 4)$ and (b4) of the P/N GaAs solar cell calculated from PC1D (a1)-(a4) and our model (b1)-(b4) with $\lambda=600 \mathrm{~nm}$. Device parameters are the same as those used in Figure 1.

be obtained directly from the profiles of $\Phi$ [Figure 2(a2 and b2)] or $F$ [Figure 2(a3 and b3)]. Again, both methods show the same results, and this agreement is held for all wavelengths (not shown), revealing that our model is technically and physically valid for the simulation of 1D SCs. In addition, we have identified an error in the value of $\Phi$ reported by PC1D (version 5.9) [see Figure 2(a2)]; it appears that $\Phi$ is calculated correctly internally by PC1D, because the values we calculate can be recovered by multiplying the PC1Dreported values by the constant $K_{\mathrm{B}} \mathrm{T} / q$ [see Figure 2(b2)].
Besides the total carrier recombination rate, $U$, detailed information on the contribution of each component can be obtained from our calculation, providing a better understanding of the role of the respective recombination types in affecting the device performance. Shown in Figure 3 are the spatial profiles of $U_{\mathrm{SRH}}, U_{\text {rad }}$, and $U_{\text {aug }}$ at $\lambda=400$, 600 , and $920 \mathrm{~nm}$, respectively, and under no electric bias $(V=0 \mathrm{~V})$. It can be seen that: (i) in such a direct bandgap material $(\mathrm{GaAs}), U_{\text {rad }}$ dominates the total recombination process; (ii) low carrier recombination rates are found in

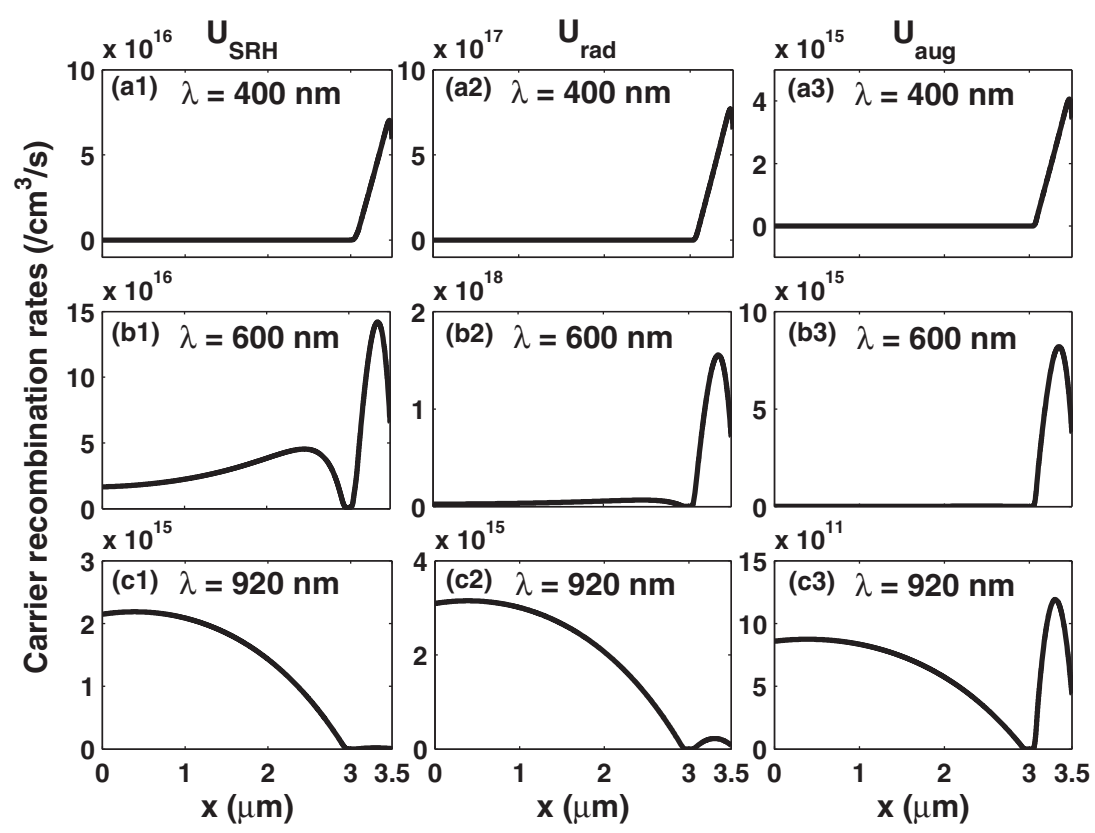

Figure 3. Profiles of $U_{\mathrm{SRH}}(\mathrm{a} 1)$, (b1), and (c1), $U_{\text {rad }}(\mathrm{a} 2)$, (b2), and (c2), and $U_{\text {aug }}(\mathrm{a} 3)$, (b3), and (c3) of the P/N GaAs solar cell calculated from our model with $\lambda=400 \mathrm{~nm}$ (a1)-(a3), $600 \mathrm{~nm}$ (b1)-(b3), and $920 \mathrm{~nm}$ (c1)-(c3), respectively. Device parameters are the same as those used in Figure 1, under no electric bias (short circuit). 
the space charge region; (iii) stronger recombination rates were found at $\lambda=600 \mathrm{~nm}$, which is due to the higher carrier concentration under stronger light injection (from the solar illumination spectrum) [28]; (iv) with a strong light absorption, the recombination profile is weighted towards the region close to the surface on the light injection side; and (v) with the light absorption weakened at long wavelengths, the solar incidence penetrates further into the cell, driving the location with maximum $U$ to move towards the rear side. The ability to extract such information on the spatial locations of recombination will be critical in the simulation of SCs that employ local field concentration elements, such as plasmonic structures.

We should indicate that the accuracy of the Beer-Lambert approximation becomes progressively less accurate with an increasing number of layers, because light interference in the waveguide(s) strongly reshapes the field distribution in each layer. A more accurate way to obtain the carrier generation profile is to include optical phase into the treatment, for example, the transfer-matrix method [40]; however, this is only valid for devices showing no noticeable transverse dependence, hence, it is not appropriate for plasmonic SCs. Having established the validity of our model in 1D, we now extend our exact EM calculations and device model to higher dimensions in the next section.

\section{2D MODELING WITH ELECTROMAGNECTIC AND ELECTRONIC CALCULATIONS}

In this section, a complete $2 \mathrm{D}$ simulation was performed for the previously discussed homojunction P/N GaAs SC (device configuration given in the inset of Figure 4). Because of the additional spatial dependence under consideration, periodic boundary conditions were used in the transverse direction for both the optical and electronic modules. In this

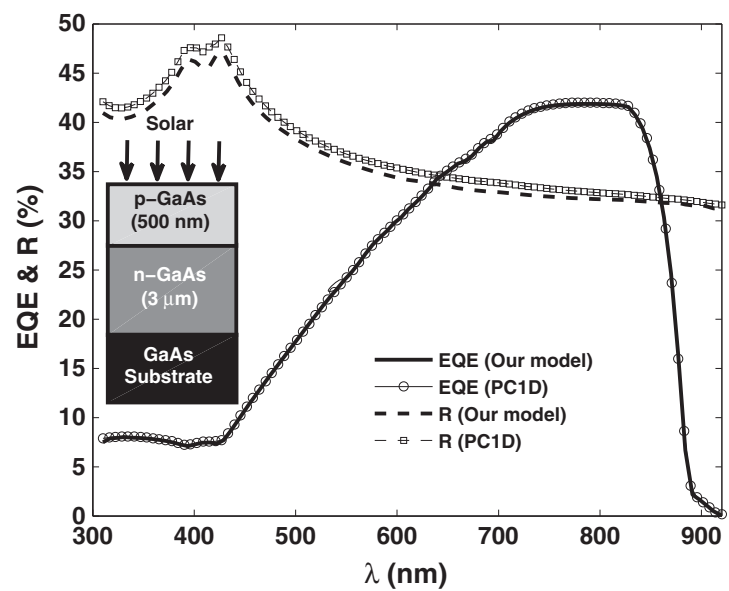

Figure 4. External quantum efficiency (EQE) and $R$ spectra of a P/N GaAs solar cell calculated from our complete 2D model and PC1D. Surface reflectivity is automatically calculated by the EM module. way, the carrier generation profile was obtained from EM calculations in 2D. Reflectivity and EQE spectra after a complete calculation (considering both optical and carrier transport responses) are given in Figure 4, where PC1D results are also shown for comparison. Small differences between the results from our 2D model and PC1D for an identical SC now emerge. This occurs because the reflectivity spectrum is estimated by considering the first several layers in PC1D instead of exactly solving the wave equations for the whole device. The device discussed here has a relatively simple configuration; therefore, such estimation does not cause the results to deviate significantly from our comprehensive $2 \mathrm{D}$ calculation, leading to only a small change to $\mathrm{EQE}$. However, more significant changes are expected with strongly confining photonic nanostructures.

Further comparison is therefore necessary for an efficient heterojunction GaAs SC (see Figure 5) [41-43], where window and BSF layers were used to prohibit the minority carrier diffusion toward the surfaces. In addition, dual-layer ARCs, consisting of $100-\mathrm{nm} \mathrm{MgF}_{2}$ (magnesium fluoride) and 50-nm ZnS (zinc sulfide), were considered to minimize surface reflection loss [43]. The parameters for this simulation are listed in Table I. However, to better match the experiments, $\tau_{p}$ in the window, emitter, and base layers was increased to $80 \mathrm{~ns}$ in the calculation of EQE to account for the photon-recycling effect [44]; correspondingly, a lower $B_{\text {rad }}$ (assumed to be $10 \%$ of its original value) was used. As indicated in [43], the photon-recycling effect actually increases the limiting efficiency achievable in GaAs SCs because, as shown in Figure 3, radiative recombination dominates the total carrier recombination process in GaAs SCs. Accounting for photon-recycling in a full 2D electromagnetic wave model is computationally demanding because the radiative recombination feeds back into the electromagnetic field. At present, our model treats photon recycling through the choice of the appropriate $B_{\text {rad }}$ coefficient, but a more complete treatment of the photonrecycling effect can be found in previous reports (e.g., [44]) and will be incorporated into our model in the future.

Our results are shown in Figure 5, which plots the spectra of current densities (a), EQE and $R$ spectra (b), internal quantum efficiency (IQE) spectrum [41] (c) as well as the $I-V$ curve (d) of the SCs. The corresponding performance parameters extracted from the $I-V$ curve are inserted into Figure 5(d), where a grid shadow $\sim 4.9 \%$ was used [41]. Compared with Figure 4, the device performance is improved significantly because of better optical and electronic design. Under our proposed complete simulation scheme, the performance of such a multi-layered SC can be accurately predicted and shows a good agreement with experiments in terms of both the optical and electronic response. The experimental data from [43] are shown in Figure 5(c and d). The difference between our prediction and the experimental result is less than $1 \%$.

An alternative simulation for the same device is conducted in PC1D, where the indices of $\mathrm{MgF}_{2}$ and $\mathrm{ZnS}$ are 1.38 and 2.25 , respectively, because the material dispersion of ARC layers could not be taken into account 

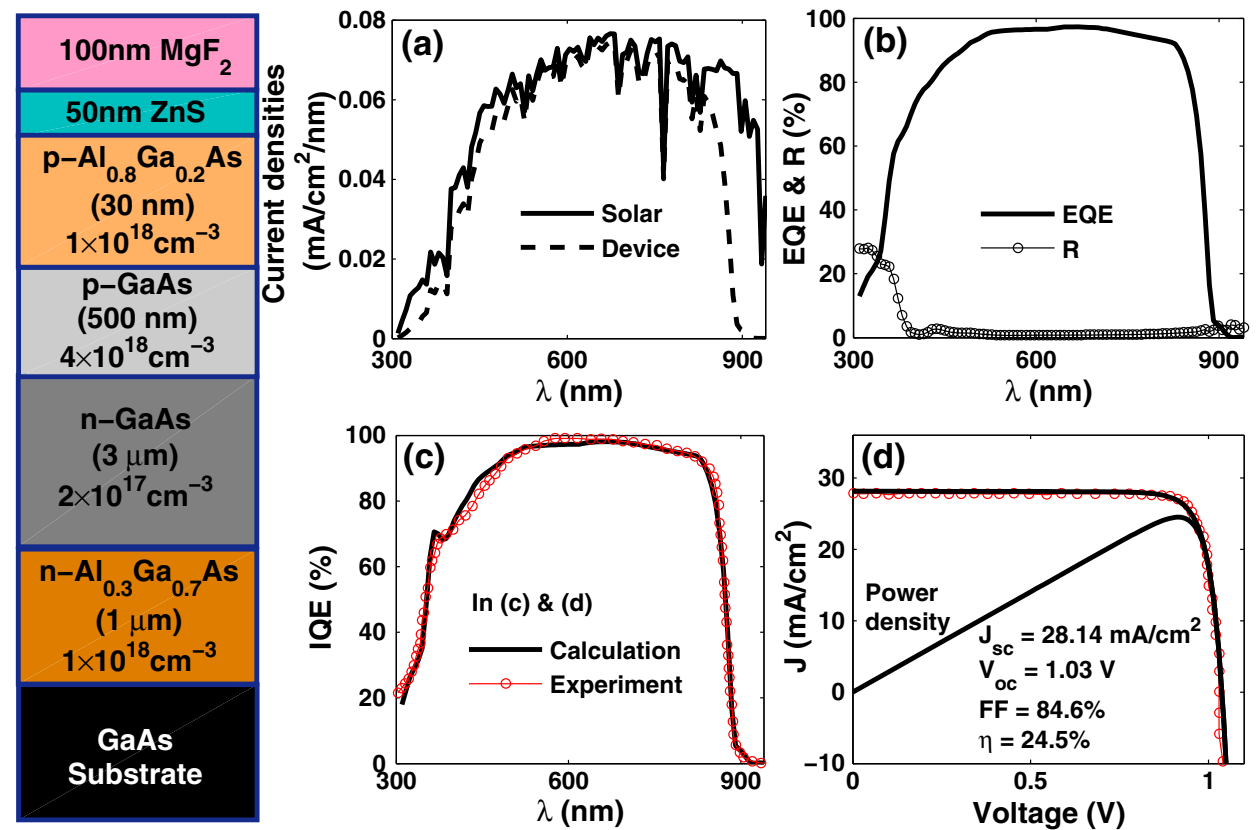

Figure 5. Current density spectrum (a), external quantum efficiency (EQE) and $R$ spectra (b), internal quantum efficiency (IQE) spectra (c) and $I-V$ curves (d) of a typical heterojunction GaAs solar cell calculated from our complete 2D model and reported from experiment (circles) [43]. The equivalent solar current density spectrum and output power density from the device are also shown in (a) and (d),

respectively. The detailed device configuration and the device performance parameters are also displayed in this figure.

straightforwardly. Calculated EQE and $R$ spectra, plotted in Figure 6(a), are obviously overestimated compared with the reported experimental results and those from our simulation, especially when $\lambda<400 \mathrm{~nm}$. We believe the discrepancy arises from two factors. First, the precise $R$ is not obtained due to the use of constant material indices for the ARC layers as well as the lack of addressing the EM response of the whole device. The second reason is that the absorption of the $\mathrm{ZnS}$ layer at short wavelengths is not taken into account [42]. The accuracy of this multi-layered PC1D simulation can be improved after the following corrections, however. The first is a reflection correction, which involves importing the $R$ spectrum obtained from our EM module into PC1D. The updated results, shown in Figure 6(b), are indeed improved but still cannot accurately reflect realistic conditions. Only by using the total loss (surface reflection loss $+\mathrm{ZnS}$ absorption) can the heterojunction GaAs SCs be modeled more accurately [see Figure 6(c)]. This example demonstrates how our exact EM calculation leads to a more accurate simulation of SCs. We would like to indicate that transfer-matrix method can also be used to perform a similar calibration for PC1D; however, as mentioned in Section 3, its applicability is limited to those systems with planar multi-layered configuration. On the contrary, our calculation is much more flexible for the simulations of various systems.
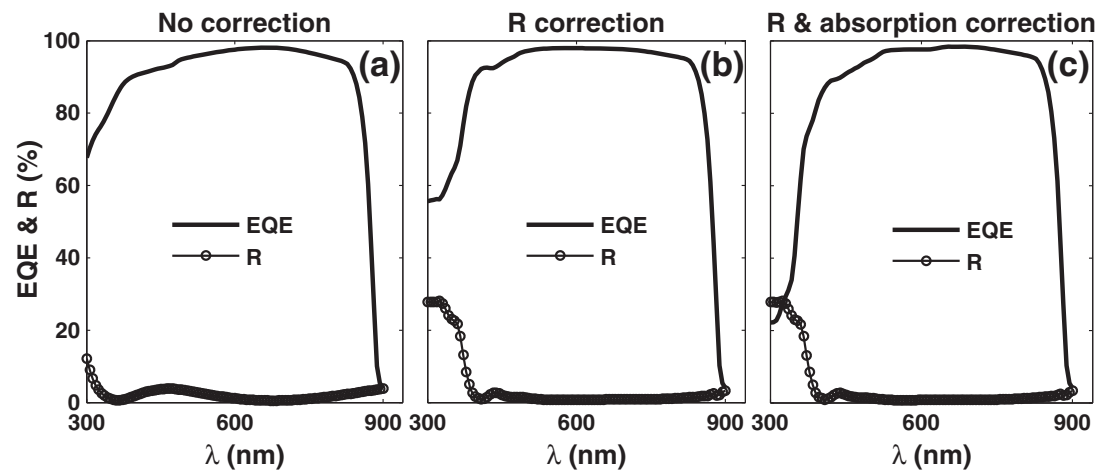

Figure 6. PC1D-calculated external quantum efficiency (EQE) and $R$ spectra for the GaAs solar cell considered in Figure 5. Here, PC1D results without correction, with $R$ correction, and with $R \& \mathrm{ZnS}$ absorption correction are given in (a), (b), and (c), respectively. 


\section{3D MODELING}

To account for non-uniform carrier generation due to the presence of plasmonic nanostructures, it is desirable but challenging for us to perform a complete 3D simulation of a realistic $\mathrm{SC}$, that is, to model both optical response and carrier transport in 3D. Nevertheless, this can be achieved in a conceptually straightforward manner with our model by including all spatial degrees of freedom. If we were to simulate the devices considered previously in $1 \mathrm{D}$ and $2 \mathrm{D}$ cases, then the 3D model outputs the same performance parameters (e.g., IQE, EQE, $J_{\mathrm{sc}}, V_{\mathrm{oc}}, \eta$, etc.) as before, and additionally provides $3 \mathrm{D}$ spatial information

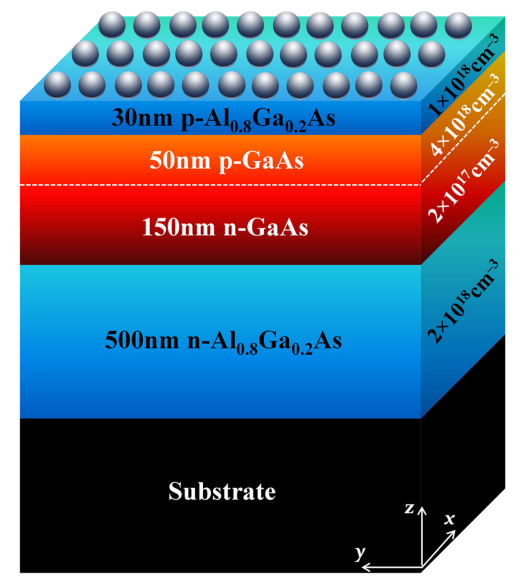

Figure 7. Schematic diagram of the plasmonic thin-film GaAs solar cells, where the physical dimension and doping concentration of each layer have been given. on many optical and electronic parameters (e.g., $n, p, \Phi, g$, $U$, etc.). One of the attractive points of the proposed complete 3D simulation is its capability to model SCs that have strong spatial dependences for the optical and electronic parameters, such as PSCs.

In this section therefore, the simulation of a thin-film heterojunction GaAs SC decorated with silver nanoparticles on top of the window layer (see Figure 7) is presented. The underlying SC structure was taken from [17], where a 500-nm n-type $\mathrm{Al}_{0.8} \mathrm{Ga}_{0.2} \mathrm{As}$ BSF layer was used. For the experiment to be accurately matched, several parameters listed in Table I have been carefully modified: $S_{n}=10^{6} \mathrm{~cm} / \mathrm{s}$, $S_{p}=10^{5} \mathrm{~cm} / \mathrm{s}, \quad \mu_{n}=100 \mathrm{~cm}^{2} / \mathrm{V} / \mathrm{s} \quad\left(\mu_{p}=10 \mathrm{~cm}^{2} / \mathrm{V} / \mathrm{s}\right)$ in the window (BSF) layer, $\mu_{p}=80 \mathrm{~cm}^{2} / \mathrm{V} / \mathrm{s}$ in the GaAs layers, $\tau_{n}$ $\left(\tau_{p}\right)=1 \mathrm{ps}$ in the window (BSF) layer, and $\tau_{n}=0.01 \mathrm{~ns}$ and $\tau_{p}=0.1 \mathrm{~ns}$ in other regions. Our simulation employed periodically arranged silver spheres instead of randomly distributed silver hemispheres due to the periodic nature of the model. A linearly polarized source (along $x$ ) was used because such a rotationally symmetric device is insensitive to the incident polarization.

Simulation results are plotted in Figure 8, which shows that under the non-plasmonic thin-film configuration, the light absorption of the device has been strongly weakened, and $R \sim 30 \%$ is observed due to the absence of ARC layers. However, if the SC is covered with a periodic array of 110-nm-diameter silver nanospheres (areal concentration $=$ $1.8 \times 10^{9} \mathrm{~cm}^{-2}$ ), the total EQE can be significantly improved [see Figure 8(c)], although EQE can also be decreased in the short-wavelength region due to the destructive interference between the incident and scattered light [45].

The overall performance improvement of the SC arising from plasmonic design can be easily verified by examining
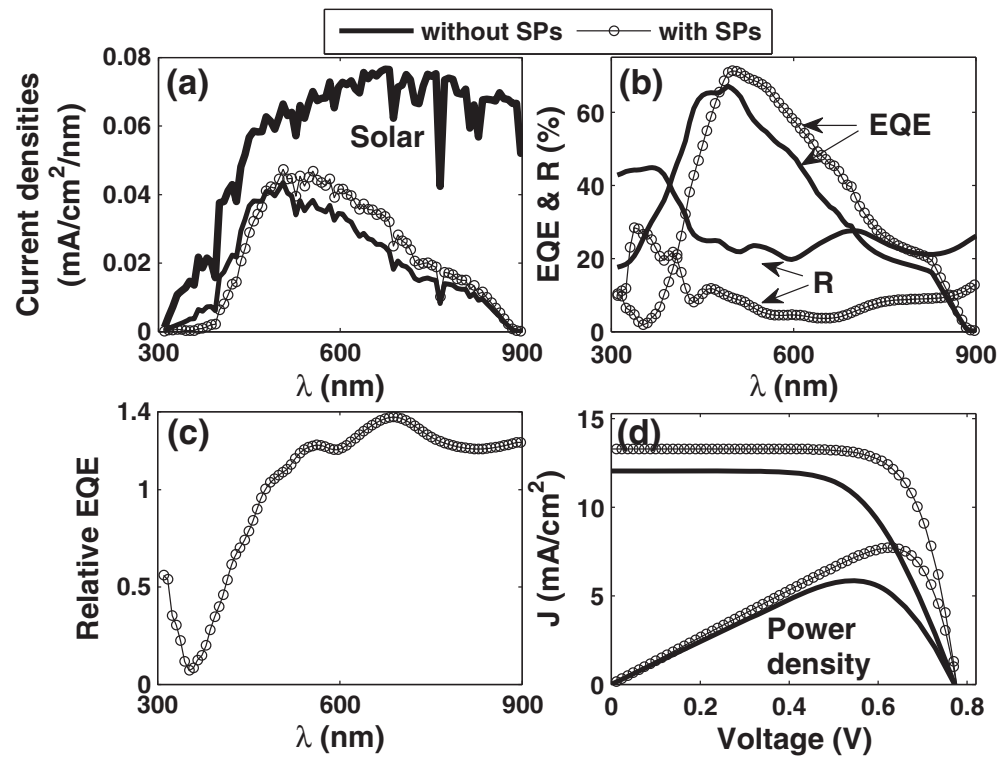

Figure 8. Current density spectra (a), external quantum efficiency (EQE) and $R$ spectra (b), relative EQE spectrum (relative to non-plasmonic case) (c), and $1-V$ curves (d) of the solar cells without (solid) and with (circles) plasmonic design. The equivalent solar current density spectrum and output power densities from the device are also shown in (a) and (d), respectively. 
(a)

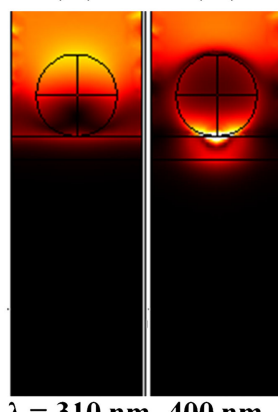

$\lambda=310 \mathrm{~nm} 400 \mathrm{~nm}$ (c)

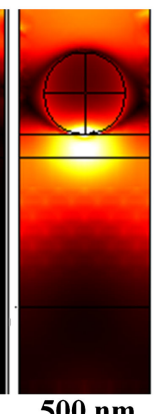

(d)

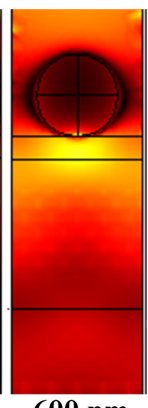

(e)

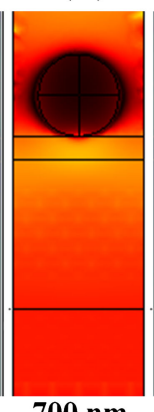

(f)

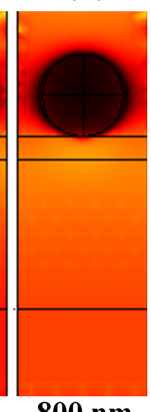

(g) Power

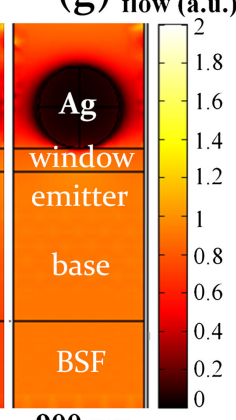

Figure 9. Power density distributions in the $y z$ plane ( $x=0$, i.e., perpendicular to the electric polarization direction) of the GaAs plasmonic solar cells. $\lambda=310,400,500,600,700,800$, and $900 \mathrm{~nm}$ in (a)-(g), respectively.

the corresponding $I-V$ response. Figure $8(\mathrm{~d})$ shows that $J_{\mathrm{sc}}$ is increased from 12 to $13.3 \mathrm{~mA} / \mathrm{cm}^{2}$ (enhancement ratio $10.2 \%$ ) after incorporating SP nanostructures. On the other hand, as discussed in [17], an extra improvement in $F F$ is achieved due to the reduced surface sheet resistance after adding the metallic nanoparticle coating. The $R_{\mathrm{S}}$ value estimated from [17] is 11 and $2 \Omega \mathrm{cm}^{2}$ before and after adding the metallic particles, whereas $R_{\mathrm{sh}}=1 \times 10^{4} \Omega \mathrm{cm}^{2}$ for both cases. Our complete 3D simulation shows that $F F$ and $\eta$ are enhanced by $18.4 \%$ and $31.2 \%$ (relative to the original values), respectively, in the PSCs. These results closely reflect the observations made in previous experiments $[17,46]$.

We finally examine the power distributions in the PSCs under various light wavelengths to study the wavelengthdependent photocurrent enhancement. The field as well as the power distributions in all spatial domains can be obtained through the EM calculation. Figure 9 shows the power density distributions in the $y z$ plane with $x=0$ for the considered PSCs. As can be seen, the incident light with short wavelengths is strongly reflected back and absorbed by the metallic particle array, leading to photocurrent loss; with further increasing $\lambda$ to exceed the plasmonic resonance [see Figure 9(c)], the incident light is strongly forwardly scattered and localized into the high-index active layers, yielding more photogenerated carriers $[45,47]$. In addition, we would like to indicate that the strong field localization in the region close to the surface due to the plasmonic design could lead to strong photo-generation close to the surface, where carriers may succumb to surface recombination. This effect can be easily examined with the use of our complete optical and electronic model in 3D. An optimal PSC design considering this carrier quenching effect will be reported in the future. These results support the need to perform comprehensive 3D simulations to fully understand the processes contributing to PSC performance improvement.

\section{CONCLUSION}

We have presented a series of comprehensive simulations of SCs including both electromagnetic and carrier transport calculations in spatial domains from 1D to 3D. The model was validated through comparison and good agreement with PC1D and existing experimental reports. Compared with conventional SC models, the careful treatment of carrier generation and the capability to study $3 \mathrm{D}$ structures make our calculations more accurate and open the possibility to analyze many complex systems. In particular, nanostructured PSCs were simulated using our 3D model, enabling a complete set of performance evaluation parameters to be determined and in close agreement with typical experimental findings.

Finally, we would like to emphasize that although GaAs SCs have been discussed here, it is straightforward to use our model to study other types of SCs (e.g., those based on various photoactive materials or system configurations). Such a scheme is also applicable for simulations of lightemitting diodes, optical detectors, and other similar optoelectronic devices.

\section{ACKNOWLEDGEMENT}

This work was supported by EU FP7 project "PRIMA" 248154.

\section{REFERENCES}

1. Nelson J. The Physics of Solar Cells. Imperial College Press: London, 2003.

2. PVCDROM (by National Science Foundation). http:// www.pveducation.org/pvcdrom

3. PC1D VERSION 5: 32-bit solar cell modeling on personal computers. Proceedings 26th IEEE Photovoltaic Specialists Conference 1997; 207. http://sourceforge. net/projects/pc1d/

4. ISE. Technology CAD overview. ISE. http://www.ise.com

5. Burgelman M, Nollet P, Degrave S. Modelling polycrystalline semiconductor solar cells. Thin Solid Films 2000; 361-362: 527-532. 
6. Froitzheim A, Stangl R, Elstner L, Kriegel M, Fuhs W. AFORS-HET: a computer-program for the simulation of hetero-junction solar cells to be distributed for public use. Proceedings of the 3rd World Conference on Photovoltaic Energy Conversion 2003; 1: 279-282.

7. Fonash S. Analysis of Microelectronic and Photonic Structures (AMPS) software was developed at Pennsylvania State University under the direction of S.J. Fonash with funding from the Electric Power Research Institute. (http://www.cneu.psu.edu/amps/)

8. Malm U, Edoff M. 2D device modelling and finite element simulations for thin-film solar cells. Solar Energy Materials and Solar Cells 2009; 93: 1066-1069.

9. Maier SA, Brongersma ML, Kik PG, Meltzer S, Requicha AAG, Atwater HA. Plasmonics - a route to nanoscale optical devices. Advanced Materials 2001; 13: 1501-1505.

10. Maier SA. Plasmonics: Fundamentals and Applications. Springer: New York, 2007.

11. Giannini V, Fernandez-Dominguez AI, Heck SC, Maier SA. Plasmonic nanoantennas: fundamentals and their use in controlling the radiative properties of nanoemitters. Chemical Reviews 2011; 111: 3888-3912.

12. Atwater HA, Polman A. Plasmonics for improved photovoltaic devices. Nature Materials 2010; 9: 205-213.

13. Catchpole KR, Polman A. Plasmonic solar cells. Optics Express 2008; 16: 21793-21800.

14. Pillai S, Catchpole KR, Trupke T, Green MA. Surface plasmon enhanced silicon solar cells. Journal of Applied Physics 2007; 101: 093105.

15. Matheu P, Lim SH, Derkacs D, McPheeters C, Yu ET. Metal and dielectric nanoparticle scattering for improved optical absorption in photovoltaic devices. Applied Physics Letters 2008; 93: 113108.

16. Akimov YA, Koh WS, Ostrikov K. Enhancement of optical absorption in thin-film solar cells through the excitation of higher-order nanoparticle plasmon modes. Optics Express 2009; 17: 10195-10205.

17. Nakayama K, Tanabe K, Atwater HA. Plasmonic nanoparticle enhanced light absorption in GaAs solar cells. Applied Physics Letters 2008; 93: 121904.

18. Eminian C, Haug FJ, Cuvero O, Niuille X, Ballif C. Photocurrent enhancement in thin film amorphous silicon solar cells with silver nanoparticles. Progress in Photovoltaics: Research and Applications 2011; 19: 260-265.

19. Beck FJ, Mokkapati S, Catchpole KR. Plasmonic light-trapping for $\mathrm{Si}$ solar cells using self-assembled, Ag nanoparticles. Progress in Photovoltaics: Research and Applications 2010; 18: 500-504.

20. Diukman I, Tzabari L, Berkovitch N, Tessler N, Orenstein M. Controlling absorption enhancement in organic photovoltaic cells by patterning Au nano disks within the active layer. Optics Express 2010; 19: A64-A71.

21. Nagel JR, Scarpulla MA. Enhanced absorption in optically thin solar cells by scattering from embedded dielectric nanoparticles. Optics Express 2010; 18: A139-A146.

22. Pala RA, White J, Barnard E, Liu J, Brongersma ML. Design of plasmonic thin-film solar cells with broadband absorption enhancements. Advanced Materials 2009; 21: 3504-3509.

23. Mokkapati S, Beck FJ, Polman A, Catchpole KR. Designing periodic arrays of metal nanoparticles for light-trapping applications in solar cells. Applied Physics Letters 2009; 95: 053115.

24. De Vlaminck I, Van Dorpe P, Lagae L, Borghs G. Local electrical detection of single nanoparticle plasmon resonance. Nano Letters 2007; 7: 703-706.

25. Li XF, Hylton NP, Giannini V, Lee KH, Ekins-Daukes NJ, Maier SA. Bridging electromagnetic and carrier transport calculations for three-dimensional modelling of plasmonic solar cells. Optics Express 2011; 19: A888-A896.

26. Comsol Multiphysics. http://www.comsol.com/

27. Sutherland JE, Hauser JR. A computer analysis of heterojunction and graded composition solar cells. IEEE Transactions on Electron Devices 1977; 24: 363-372.

28. Solar irradiance. http://rredc.nrel.gov/solar/spectra/ am1.5/ASTMG173/ASTMG173.html

29. Raida Z, Cernohorsky D, Gala D, et al. Electromagnetic waves microwave technique. (Multimedia Textbook, http://www.urel.feec.vutbr.cz/ raida/multimedia/ index.php)

30. Bhattacharya P. Semiconductor Optoelectronic Devices (2 ${ }^{\text {nd }}$ ed). Prentice Hall: New Jersey, 1997.

31. Debbar N, Biswas D, Bhattacharya P. Conduction-band offsets in pseudomorphis $\operatorname{In}_{x} \mathrm{Ga}_{1-x} \mathrm{As} / \mathrm{Al}_{0.2} \mathrm{Ga}_{0.8} \mathrm{As}$ quantum wells $(0.07 \leq \times \leq 0.18)$ measured by deeplevel transient spectroscopy. Physical Review B 1989; 40: 1058-1063.

32. Anderson RL. Germanium-gallium arsenide heterojunction. IBM Journal of Research and Development 1960; 4: $283-287$.

33. Palik ED. Handbook of Optical Constants of Solids. Academic Press: Orlando, 1985.

34. Luxpop, thin film and bulk index of refraction and photonics calculations. http://www.luxpop.com/

35. Filmetrics. http://www.filmetrics.com/refractive-indexdatabase $/ \mathrm{ZnS}+-+$ Cubic

36. Lowney JR, Bennett HS. Majority and minority electron and hole mobilities in heavily doped GaAs. Journal of Applied Physics 1991; 69: 7102-7110.

37. Bennett HS. Majority and minority electron and hole mobilities in heavily doped gallium aluminum 
arsenide. Journal of Applied Physics 1996; 80: 3844-3853.

38. Aspnes DE, Kelso SM, Logan RA, Bhat R. Optical properties of $\mathrm{Al}_{x} \mathrm{Ga}_{1-x}$ As. Journal of Applied Physics 1986; 60: 754-767.

39. NSM archive-physical properties of semiconductors. http://www.ioffe.ru/SVA/NSM/Semicond/

40. Li XF, Yu SF. Modeling of Rabi splitting in quantum well microcavities using time-dependent transfer matrix method. Optics Express 2008; 17: 19285-19290.

41. Tobin SP, Vernon SM, Bajgar C, Wojtczuk SJ, Melloch MR, Keshavarzi A, Stellwag TB, Venkatensan S, Lundstrom MS, Emery KA. Assessment of MOCVDand MBE-grown GaAs for high-efficiency solar cell applications. IEEE Transactions on Electron Devices 1990; 37: 469-477.

42. Tobin SP, Vernon SM, Bajgar C, Geoffroy LM, Keavney CJ, Sanfacon MM, Haven VE. Device processing and analysis of high efficiency GaAs cells. Solar Cells 1988; 24: 103-115.
43. Tobin SP, Vernon SM, Wojtczuk SJ, Bajgar C, Sanfacon MM, Dixon TM. Advances in high-efficiency GaAs solar cells. Photovoltaic Specialists Conference 1990; 1: $158-162$.

44. Durbin SM, Gray JL. Numerical modeling of photon recycling in solar cells. IEEE Transactions on Electron Devices 1994; 41: 239-245.

45. Lim SH, Mar W, Matheu P, Derkacs D, Yu ET. Photocurrent spectroscopy of optical absorption enhancement in silicon photodiodes via scattering from surface plasmon polaritons in gold nanoparticles. Journal of Applied Physics 2007; 101: 104309.

46. Shu GW, Liao WC, Hsu CL, Lee JY, Hsu IJ, Shen JL, Yang MD, Wu CH, Lee YC, Chou WC. Enhanced conversion efficiency of GaAs solar cells using Ag nanoparticles. Advanced Science Letters 2010; 3: 368-372.

47. Spinelli P, Hebbink M, De Waele R, Black L, Lenzmann F, Polman A. Optical impedance matching using coupled plasmonic nanoparticle arrays. Nano Letters 2011; 11: 1760-1765. 